\title{
An Interval Lattice Model for Grid Resource Searching
}

\author{
Wen Zhou ${ }^{1}$, Zongtian Liu ${ }^{1}$, and Yan Zhao ${ }^{2}$ \\ ${ }^{1}$ School of Computer Engineering and Science, Shanghai University, \\ ${ }^{2}$ Sydney Institute of Language and Commerce, Shanghai University, \\ Shanghai, P.R. China, 200072 \\ \{zhouwen, ztliu, zhaoyan87\}@shu.edu.cn
}

\begin{abstract}
In practice the information mostly expressed in interval form. Formal Concept Analysis can not deal with the interval information. So the research on interval lattice is an important task. In the paper, an interval lattice model is proposed. Then a Grid resource matching algorithm through the interval lattice is presented. In the end of the paper, experimental results show that the construction algorithm has reasonable performance on the complexity.
\end{abstract}

Keywords: interval concept lattice, interval FCA, Grid resource searching.

\section{Introduction}

Formal Concept Analysis (FCA) is a data analysis technique based on the ordered lattice theory firstly introduced by Wille [1]. It defines formal contexts to represent relations between objects and attributes and interprets the corresponding concept lattice.

To deal with the uncertain and vague information in practice, the interval analysis may be a better method. There are many researchers study in interval number such as [2-5]. Burusco et al introduce the interval valued fuzzy data from the view of FCA who produce a model of L-fuzzy concept theory to deal with interval valued fuzzy data from the point of view of FCA at the first time [6]. It uses some results of multisets and expertons and requires a complete chain to conduct the interval value within $[0,1]$ to form the super-sub relation between two concepts to form lattice.

Scaling [7] is used to generate concept lattice of the formal context with nominal attributes. Inspired by which, interval scaling is proposed to process interval attribute. Then, based on the interval lattice to manage the resource in Grid, a Grid resource matching algorithm is introduced. The rest of the paper is organized as follows. Section 2 introduces Interval Formal Concept Analysis Model. Section 3 presents the scale algorithm. Section 4 discusses the resource matching algorithms, the experiment is in Section 5 and section 6 concludes.

\section{An Interval Formal Concept Analysis Model}

This section produce the Interval Formal Concept Analysis (IFCA) whose core data structure is interval lattice, which incorporates attribute decomposing based interval attribute scaling to make FCA has the capacity of representing interval information. 
An interval $[a, b]$ is the set of all real numbers $\{x: a \leq x \leq b\}$. A natural definition of arithmetic for intervals, represented as pairs of real numbers[8]. The interval [a, b] with $a=b$ is called degenerate interval which degenerate to a real number a. interval decomposition denoted as $\odot$. There are two rules in decomposition process:

1. The degenerate intervals do not participate in the decomposition process.

2. The decomposition process does not generate degenerate intervals.

Firstly the two intervals decomposition process is analyzed. Both $\left[a_{1}, b_{1}\right]$ and $\left[a_{2}, b_{2}\right]$ are not degenerate intervals. Without missing the generality, we assume $a_{1} \leq a_{2}$.

$\left[a_{1}, b_{1}\right] \odot\left[a_{2}, b_{2}\right]=I_{1}, I_{2}, I_{3}=\left[a_{1}, a_{2} \wedge b_{1}\right],\left[a_{2},\left(a_{2} \vee b_{1}\right) \wedge b_{2}\right],\left[\left(a_{2} \vee b_{1}\right) \wedge b_{2}, b_{1} \vee b_{2}\right]$

If the interval $I_{i}=\left[a^{-}, a^{+}\right](\mathrm{i}=1,2,3)$ with $a^{-}=a^{+}\left(a^{-}\right.$and $a^{+}$are the lower bound and the upper bound of $I_{i}$ respectively), it does not satisfy rule 2, and will be deleted from the result. There exists eight conditions of $\left[a_{1}, b_{1}\right]$ and $\left[a_{1}, b_{2}\right]$ as show in Table 1 .

Table 1. The decomposition condition table of two intervals

\begin{tabular}{|c|c|c|c|c|c|}
\hline Type & & onditi & & {$\left[a_{1}, b_{1}\right] \odot\left[a_{2}, b_{2}\right]$} & Example \\
\hline 1 & & $\mathrm{~B} 1$ & - & {$\left[a_{1}, b_{1}\right]$} & {$[2,5] \odot[2,5]=[2,5]$} \\
\hline 2 & A1 & B2 & $\mathrm{C} 3$ & {$\left[a_{1}, b_{1}\right],\left[b_{1}, b_{2}\right]$} & {$[2,8] \odot[2,12]=[2,8],[8,12]$} \\
\hline 3 & & B3 & - & {$\left[a_{1}, b_{2}\right],\left[b_{2}, b_{1}\right]$} & {$[2,8] \odot[2,5]=[2,5],[5,8]$} \\
\hline 4 & & B1 & $\mathrm{C} 3$ & {$\left[a_{1}, a_{2}\right],\left[a_{2}, b_{2}\right]$} & {$[2,6] \odot[3,6]=[2,3],[3,6]$} \\
\hline 5 & & & $\mathrm{C} 1$ & {$\left[a_{1}, b_{1}\right],\left[a_{2}, b_{2}\right]$} & {$[2,4] \odot[4,6]=[2,4],[4,6]$} \\
\hline 6 & $\mathrm{~A} 2$ & B2 & $\mathrm{C} 2$ & {$\left[a_{1}, b_{1}\right],\left[a_{2}, b_{2}\right]$} & {$[2,3] \odot[4,6]=[2,3],[4,6]$} \\
\hline 7 & & & $\mathrm{C} 3$ & {$\left[a_{1}, a_{2}\right],\left[a_{2}, b_{1}\right],\left[b_{1}, b_{2}\right]$} & {$[2,5] \odot[4,7]=[2,4],[4,5],[5,7]$} \\
\hline 8 & & B3 & - & {$\left[a_{1}, a_{2}\right],\left[a_{2}, b_{2}\right],\left[b_{2}, b_{1}\right]$} & {$[2,11] \odot[5,9]=[2,5],[5,9],[9,11]$} \\
\hline
\end{tabular}

The condition $A 1$ and $A 2$ denote $a_{1}=a_{2}, a_{1}<a_{2}$ respectively, $B 1, B 2$ and $B 3$ denote $b_{1}=b_{2}, b_{1}<b_{2}$, and $b_{1}>b_{2}$, and $C 1, C 2, C 3$ denote $b_{1}=a_{2}, b_{1}<a_{2}$ and $b_{1}>a_{2}$ respectively.

The information table $(G, M, R)$ (the relation of $R(g, m)((g \in G, m \in M)$ has the value of $u(g, m))$ can be represented as a cross-table shown in Table 2. The information table has three objects representing three Grid resources, namely $C 1, C 2$ and C3. In addition, it also has three attributes, "CPU capacity" (A), "Memory Size" (B), "Resource Price" (C) and "Working Time" (D) representing four properties of the Grid resource. The relation between an object and an attribute is represented by a membership value expressed by interval.

Table 2. Information table of interval

\begin{tabular}{ccccc}
\hline & $\mathrm{A}$ & $\mathrm{B}$ & $\mathrm{C}$ & $\mathrm{D}$ \\
\hline C1 & {$[0-2.8]$} & {$[0-512]$} & {$[200-10000]$} & {$[9-12]$} \\
C2 & {$[0-8.8]$} & {$[0-256]$} & {$[300-10000]$} & {$[20-24]$} \\
C3 & {$[0-1.4]$} & {$[0-1024]$} & {$[500-10000]$} & {$[5-11]$} \\
\hline
\end{tabular}


Interval attribute decomposition is for $m(m \in M)$, the intervals $u(g, m)$ of $R(g$, $m)$ in $(G, M, R)$ is decomposed as $W_{m}$. The information table can be transfer to interval context by interval attribute scaling.

Definition 1: An interval attribute scaling. After interval attribute decomposition the information table $(G, M, R)$ is extended to $\left(G, M,\left(W_{m}\right)_{m} \in_{M}, R, I\right)$, each $W_{m}$ is a set of decomposed intervals of $\mu(g, m)$ for the attribute $m$ ( $m \in M, g \in G$ ), $(g, m, w) \in I$ if and only if there exists $w \subseteq \mu(g, m)\left(w \in W_{m}, \mu(g, m)\right.$ is the value of $R(g, m)$ of information table $(G, M, R))$.

Definition 2: Interval formal context. It is a tuple $\mathrm{K}:=\left(G, M,\left(W_{m}\right)_{m \in M}, I\right)$ where $G$ is a set of objects, $M$ a set of attributes, and $I \subseteq G \times\left\{(m, w) \mid m \in M, w \in W_{m}\right\}$, a relation with $\left(g, m, w_{1}\right) \in I,\left(g, m, w_{2}\right) \in I \Rightarrow w_{1}=w_{2} \cdot(g, m, w) \in I$ is read "object $g$ has value $w$ for attribute $m$ ".

The interval formal context is as shown in Table 3 which is obtained from Table 2 by interval attributer scaling after attribute decomposition.

Table 3. Interval formal context

\begin{tabular}{cccccccccccccc}
\hline $\mathrm{M}$ & \multicolumn{1}{c}{$\mathrm{A}$} & \multicolumn{1}{c}{$\mathrm{B}$} & \multicolumn{1}{c}{$\mathrm{C}$} & \multicolumn{1}{c}{$\mathrm{D}$} \\
$\mathrm{W}$ & $\mathrm{A}_{1}$ & $\mathrm{a}_{2}$ & $\mathrm{a}_{3}$ & $\mathrm{~b}_{1}$ & $\mathrm{~b}_{2}$ & $\mathrm{~b}_{3}$ & $\mathrm{c}_{1}$ & $\mathrm{c}_{2}$ & $\mathrm{c}_{3}$ & $\mathrm{~d}_{1}$ & $\mathrm{~d}_{2}$ & $\mathrm{~d}_{3}$ & $\mathrm{~d}_{4}$ \\
\hline $\mathrm{C} 1$ & 1 & 1 & 0 & 1 & 1 & 0 & 1 & 1 & 1 & 0 & 1 & 1 & 0 \\
$\mathrm{C} 2$ & 1 & 1 & 1 & 1 & 0 & 0 & 0 & 1 & 1 & 0 & 0 & 0 & 1 \\
$\mathrm{C} 3$ & 1 & 0 & 0 & 1 & 1 & 1 & 0 & 0 & 1 & 1 & 1 & 0 & 0 \\
\hline
\end{tabular}

Definition 3: for $A \subseteq G$, there are two mapping:

$$
\begin{aligned}
& A^{\prime}:=\left\{w \in W_{m} \mid \forall g \in A, m \in M,(g, m, w) \in I\right\} \\
& B^{\prime}:=\left\{g \in G \mid \forall w \in W_{m}, m \in B,(g, m, w) \in I\right\}
\end{aligned}
$$

Definition 4: Interval formal concept. $C(A, B)$ is called an interval formal concept of $K:=(G, M, W, I)$ if and if only $A \subseteq G, B\left(M_{B}, W_{B}\right), M_{B} \subseteq M$, $W_{m}(B)=\left\{\cup w_{m} \mid w_{m} \in B\right\}, A^{\prime}=B$ and $B^{\prime}=A, A$ and $B$ are called the extent and intent of $C$ respectively.

Definition 5: Interval lattice. The concepts of a given context are naturally ordered by the partial relation defined by $C_{1}\left(A_{1}, B_{1}\right) \leq C_{2}\left(A_{2}, B_{2}\right): \Leftrightarrow A_{1} \subseteq A_{2} \Leftrightarrow W_{m}\left(B_{2}\right) \subseteq W_{m}\left(B_{1}\right)$ $\left(W_{m} \in B_{2}\right)$. The ordered set of all interval formal concepts of $\mathrm{K}:=(G, M, W, I)$ is denoted by $\underline{\mathrm{B}}(G, M, W, I)$, and is called the interval concept lattice of $(G, M, W, I)$. Without ambiguousness, the interval concept lattice is called concept lattice or lattice. 


\section{The Interval Lattice Construction Principle}

The attribute decomposition is the base of attribute scale to form the interval context after the attribute decomposition. The attribute decomposition algorithm is extended from the two interval decomposition according to the definition equation (1). According to the results in Table 1, the decomposition process of two intervals is shown in Table 4. Attribute decomposition of the context is based on the method of two-interval-decomposition.

Table 4. The two interval decomposition following the condition in Table 1

\begin{tabular}{|c|c|c|c|c|c|c|}
\hline \multirow{2}{*}{ Type } & \multirow{2}{*}[a_{1},b_{1}]{$\odot\left[a_{2}, b_{2}\right]$} & \multirow{2}{*}{$\mathrm{W}_{\mathrm{m}}$} & \multicolumn{2}{|c|}{ Return } & \multirow{2}{*}{$\begin{array}{c}\text { Return } \\
\text { No. }\end{array}$} & \multirow{2}{*}{ Flag } \\
\hline & & & $r_{1}$ & $\mathrm{r}_{2}$ & & \\
\hline 1 & {$\left[\mathrm{a}_{1}, \mathrm{~b}_{1}\right]$} & $W_{m}$ & {$\left[a_{1}, b_{1}\right]$} & - & 1 & true \\
\hline 2 & {$\left[\mathrm{a}_{1}, \mathrm{~b}_{1}\right],\left[\mathrm{b}_{1}, \mathrm{~b}_{2}\right]$} & $W_{m}=W_{m}+\left[\mathrm{a}_{1}, \mathrm{~b}_{1}\right]$ & {$\left[\mathrm{b}_{1}, \mathrm{~b}_{2}\right]$} & - & 1 & true \\
\hline 3 & {$\left[\mathrm{a}_{1}, \mathrm{~b}_{2}\right],\left[\mathrm{b}_{2}, \mathrm{~b}_{1}\right]$} & $W_{m}$ & {$\left[a_{1}, b_{2}\right]$} & {$\left[\mathrm{b}_{2}, \mathrm{~b}_{1}\right]$} & 2 & true \\
\hline 4 & {$\left[\mathrm{a}_{1}, \mathrm{a}_{2}\right],\left[\mathrm{a}_{2}, \mathrm{~b}_{2}\right]$} & $W_{m}=W_{m}+\left[\mathrm{a}_{1}, \mathrm{a}_{2}\right]$ & {$\left[\mathrm{a}_{2}, \mathrm{~b}_{2}\right]$} & - & 1 & true \\
\hline 5 & {$\left[\mathrm{a}_{1}, \mathrm{~b}_{1}\right],\left[\mathrm{a}_{2}, \mathrm{~b}_{2}\right]$} & $W_{m}=W_{m}+\left[\mathrm{a}_{1}, \mathrm{~b}_{1}\right]$ & {$\left[\mathrm{a}_{2}, \mathrm{~b}_{2}\right]$} & - & 1 & false \\
\hline 6 & {$\left[\mathrm{a}_{1}, \mathrm{~b}_{1}\right],\left[\mathrm{a}_{2}, \mathrm{~b}_{2}\right]$} & $W_{m}=W_{m}+\left[\mathrm{a}_{1}, \mathrm{~b}_{1}\right]$ & {$\left[\mathrm{a}_{2}, \mathrm{~b}_{2}\right]$} & - & 1 & false \\
\hline 7 & {$\left[a_{1}, a_{2}\right],\left[a_{2}, b_{1}\right],\left[b_{1}, b_{2}\right]$} & $W_{m}=W_{m}+\left[\mathrm{a}_{1}, \mathrm{a}_{2}\right]$ & {$\left[\mathrm{a}_{2}, \mathrm{~b}_{1}\right]$} & {$\left[\mathrm{b}_{1}, \mathrm{~b}_{2}\right]$} & 2 & true \\
\hline 8 & {$\left[\mathrm{a}_{1}, \mathrm{a}_{2}\right],\left[\mathrm{a}_{2}, \mathrm{~b}_{2}\right],\left[\mathrm{b}_{2}, \mathrm{~b}_{1}\right]$} & $W_{m}=W_{m}+\left[\mathrm{a}_{1}, \mathrm{a}_{2}\right]$ & {$\left[\mathrm{a}_{2}, \mathrm{~b}_{2}\right]$} & {$\left[\mathrm{b}_{2}, \mathrm{~b}_{1}\right]$} & 2 & true \\
\hline
\end{tabular}

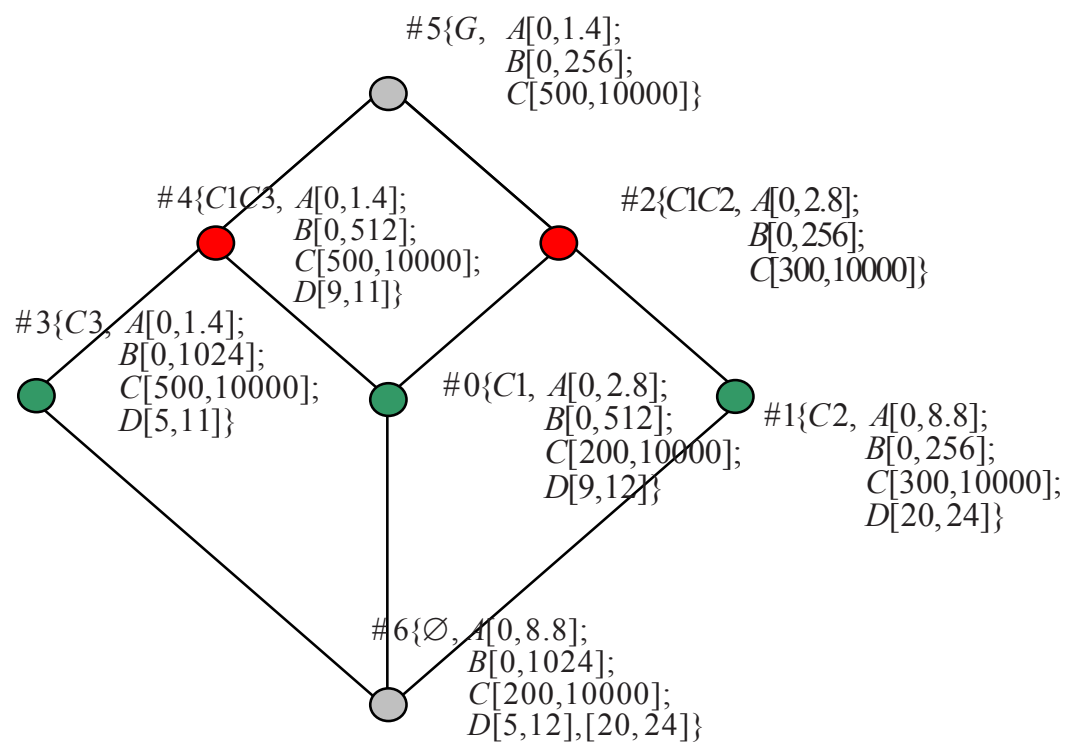

Fig. 1. The interval lattice 
By attribute decomposition, the decomposed attribute set $\mathrm{W}$ is obtained. According to the attribute scale definition the interval context is generated as an example shown in Table 1. The lattice is generated as Figure 1 from context shown as Table 3.

\section{The Resource Searching Algorithm in Grid Environment}

In Grid environment, one issue is how to effectively manage the Grid resource. Another is when a resource request is submitted, how to find out the fitful Gird resource to satisfy the request. The request is given out in the form of interval set [9]. This is our motivation to propose the interval lattice model and extend the FCA to IFCA. Using IFCA as the method to solve the two issues, interval lattice as tools to manage the Grid resource and based on which the task of resource searching according to the resource request is carried out.

According to the type of the request, we design two algorithms for search fitful Grid resources. One is bottom-up algorithm which is much quicker for the complex requests with many attributes than simple ones as shown in Figure 2. The other is topdown algorithm which is quicker for the simple request with few attributes than complex ones. The judgeRequest algorithm is to judge if a node in lattice match the request. For the reason of page limit, we only introduce the first method in this paper.

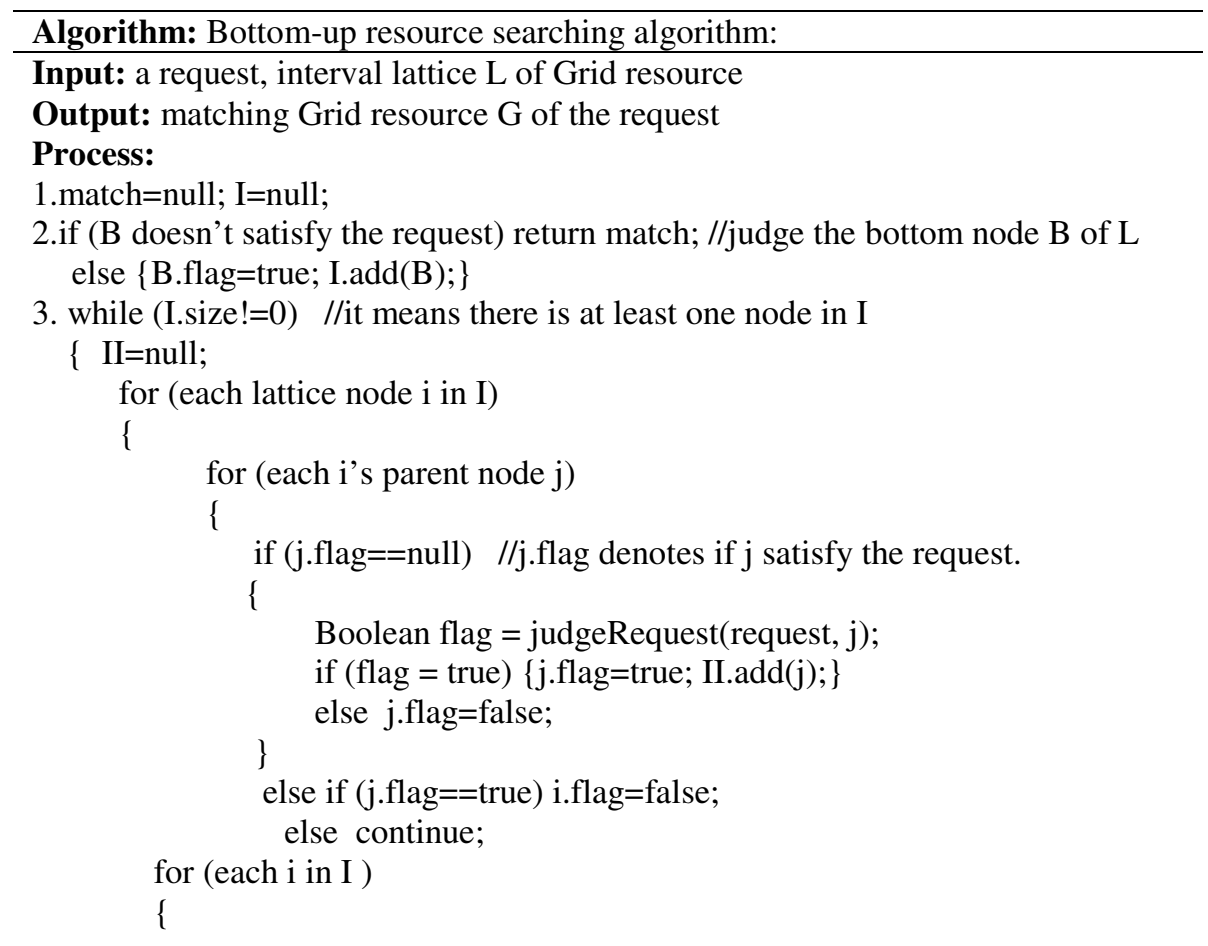

Fig. 2. The Bottom-up resource searching algorithm 


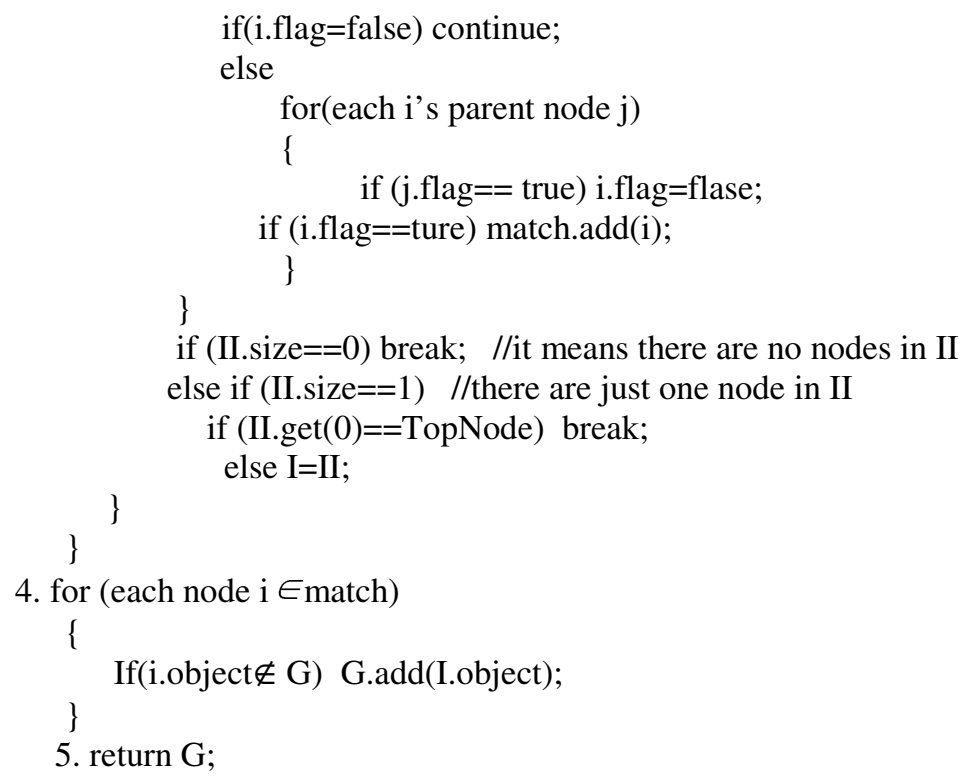

Fig. 2. (Continued)

\section{Experiments and Discussion}

Adopting the random data as the test data, all the algorithms realized with Java running on PC (CPU PIV 2.8G, memory 512M) within Windows XP environment. To improve the reality of the experiments, each of experiments with different parameters carries on five randomly generated data and take the average values of results of five times of experiments as the final result.

First, the spatial consuming of incremental lattice construction algorithm are tested. Figure 3 expresses the spatial complexity. Curve 1 and Curve 2 show the numbers of lattice nodes changing according to the different number of objects and the different numbers of attributes respectively. The horizontal ordinate indicates both the numbers of objects and attribute are respectively $\{10,20,30,40,50,60\}$. The vertical ordinate

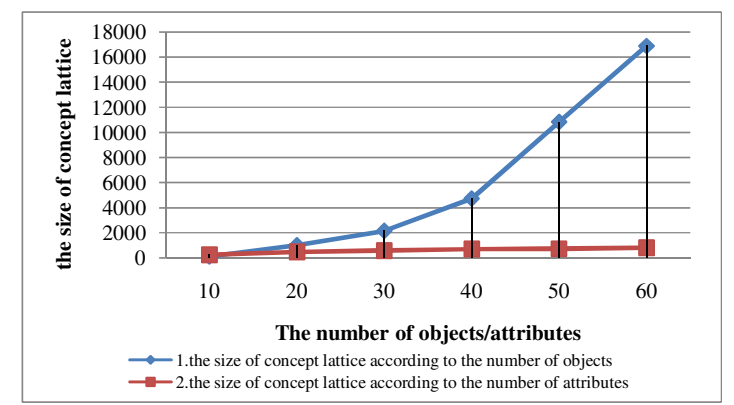

Fig. 3. Relations between the size of lattice and the numbers of both objects and attributes 
indicates size of lattice. With the ascending of object numbers, the size of the lattice has exponential ascending tendency. The influence of attribute number has very slight impact on the size of lattice.

The curves confirm the results that the numbers of objects have big influence on the lattice both in the size and the numbers of attributes haven't so strong impact on the result lattice. Fuzzy lattice spatial consuming character is different. In fuzzy FCA [10], the numbers of objects has slight influence on lattice size, but the attribute numbers has big impact on it.

\section{Conclusion}

In this paper, interval lattice (IL) model produced extend FCA to IFCA which can deal with the real interval values in practice. It is different from the classical FCA. The future research include appling this interval lattice as the Grid resource management tools and using the resource searching algorithm as the resource match tools in the real Grid enviornments to using the real data of Grid environment to further test the model and algorithms we proposed.

\section{Acknowledgments}

The work presented in this paper is supported partially by National Science Foundation of China (reference number: NSFC 60275022 and NSFC 60575035).

\section{References}

1. Wille, R.: Restructuring lattice theory: an approach based on hierarchies of concepts, Reidel, Dordrecht (1982)

2. Young, R.C.: The algebra of many-valued quantities. Annals of Mathematics 104 (1931) 260-290

3. Markov, S., Okumura, K.: The Contribution of T. Sunaga to Interval Analysis and Reliable Computing. Kluwer Academic Publishers (1999)

4. Moore, R.E.: Interval analysis. Prentice-Hall Englewood Cliffs, NJ (1966)

5. Fernandez, A.J., Hill, P.M.: An interval lattice-based constraint solving framework for lattices. 4th International Symposium on Functional and Logic Vol. 1722 (1999) 194-208

6. Burusco, A., Fuentes-González, R.: The study of the interval-valued contexts. Fuzzy Sets and Systems 121 (2001) 439-452

7. Prediger, S., Stumme, G.: Theory-driven logical scaling. International Workshop on Description Logics, Vol. 22 (1999)

8. Moore, R.E., Bierbaum, F.: Methods and Applications of Interval Analysis. Soc for Industrial \& Applied Math (1979)

9. Buyya, R.: Economic-based Distributed Resource Management and Scheduling for Grid Computing. Vol. Ph.D.. Monash University Australia (2002)

10. Qiang, Y., Liu, Z.T., al., e.: Research on fuzzy concept lattice in knowledge discovery and a construction algorithm. Acta Electronica Sinica 33 (2005) 350 353 